\title{
Contextual Coaching: Leveraging Context for Alignment in the System
}

\author{
Michael Valentine \\ Atlanta, USA
}

\begin{abstract}
Contextual coaching is a process that emphasizes the importance of an organization's environment or context on leadership development work. It recognizes that certain attributes in an organization's context like strategy, the organization's attitude towards development, prevailing leadership style or feedback quality can have disproportionate impact on coaching outcomes and the sustainability of coaching work. Further, the process posits that achieving alignment between the contextual factors most relevant to the coaching work, the developmental objectives of the client and organizational goals and expectations, will result in better outcomes for both the client and the sponsoring firm (Gorrell \& Hoover 2009). Although it is accepted that context surrounds all coaching work (Johns, 2006), there is an absence of empirical research on how it influences coaching effectiveness and sustained behavior change in organizations. This paper examines the perceived presence of contextual factors in an organization, considers how coaches incorporate them in practice, and suggests a framework to enhance coaching outcomes. The multi-phased qualitative/quantitative study utilizes the coach's perspective to identify which contextual factors they most incorporate into their work and which factors they believe are most influential in promoting the sustainability of behavior change by the coaching client.
\end{abstract}

Keywords: Executive Coaching, System Coaching, Contextual Coaching

\section{Introduction}

This theory-based research study examines the extent to which organizational context influences coaching outcomes and the sustained behavior change of a coaching client. The study uses the perspective of the coach to assess the influence of context on their ability to achieve desired coaching outcomes, and the coaching client's ability to sustain agreed upon change objectives resulting from the coaching relationship.

The organizational interest in leveraging contextual knowledge is obvious. Firms that invest in coaching as a developmental intervention 
generally have two compelling needs they wish to meet: first, they wish to ensure the investment made in the coaching-focused development yields a return through resulting desired behavior change in the individual or group that participates in the coaching; and second, the organization wishes to learn from each engagement to improve the overall developmental environment for future investments and activities (Gorrell, et al., 2009). It is clear, then, that a knowledge of the system-level factors that inhibit or support coaching work, and the ability of an individual or group to sustain behavior change over time, would be useful in creating the conditions that are most conducive to accelerated organizational learning and development. Further, this knowledge would allow the organization to better guide the alignment of the investment with overall business objectives and expectations.

One approach - that fosters understanding of these factors and examines the potential importance of the various contextual elements on developmental outcomes - assesses the experience of internal and external coaches working in the organization. Coaches hold unique positionality by virtue of their exposure to the many "touch points" in the organization they interact with during their work. It is not unusual for a coach to speak with numerous stakeholders to obtain feedback on behalf of a coaching client. These stakeholders, while providing feedback relevant to the development work, often will offer their perspective on organizational attributes that might support or challenge the developmental goals of the client. Each of these interactions, therefore, provides a chance to collect data, at the systems level, regarding the relationship between the context of the organization and development. After multiple conversations or engagements in the same system, a coach will have the opportunity to uncover themes and patterns in that system, that may have implications for the overall developmental environment. To the extent the coach understands and incorporates the knowledge gained from these patterns, it may inform approaches to development that are more effective in that organization.

\section{Literature Review}

Context influences many aspects of organizational life. Relevant studies using the narrow term "organizational context" in the title have examined its influence on organizational behavior (Johns, 2006), leadership (Porter, et al., 2006), organizational creativity (Shalley, et al., 2004), work team effectiveness (Doolen, et al., 2003), quality (Glasgow, et al., 2013) and Total Quality Management (TQM) (Sila, 2007), and its influence on turnover and job satisfaction (Parzinger, et al., 2012). Studies using the broader term of 
organizational culture yield additional support for the link between context/culture factors and organizational outcomes (Gregory, et al., 2009) for example. At the individual or group level the relationship between behavior change and context is complex; however, there is established research on how it influences the formation of attitudes (Eagly, et al., 1993) and can even help predict behavior change (Fishbein, et al., 2010). Despite these studies, there still is a dearth of extensive work on the relationship between context and coaching or leadership behavior change (Porter, et al., 2006). This point is made clearly by Funder (2001) in discussing contemporary personality research:

For all the arguments that the situation (context) is all important ... little is empirically known or even theorized about how situations influence behavior, or what the basic kinds of situations are (or alternatively, what variables are useful in comparing one situation with another). (Funder, 2001, p. 211)

An example of how additional examination of the topic would benefit the field can be found in the contextual coaching framework described in one prominent model. The model identifies ten broad components that represent organizational alignment factors that a coach should account for and incorporate into their work with a coaching client. Though the framework does provide guidance on how a coach should consider these factors, it is not specific on how to align the factors. The framework does not offer a precise measurement methodology to instruct a coach on how factors should be weighted or how factor importance may shift with different contextual profiles or differences in developmental objectives. The framework's ten factors are grouped into five dyads: Strategy/Structure, Culture/Communications, Talent Systems/Talent Solutions, Development/Dynamics (Gorrell, et al., 2009). They are intended to act as a filter for the coach while they work with their client on adjusting habits, behaviors, and skills. The dyads account for:

- Strategy - Structure: Strategy impact on leader and relationships within organizational structure

- Culture - Communication: How culture presents barriers and opportunities, and how communication style impacts success

- Talent Systems - Talent Solutions: How organizational processes impact development opportunities

- Development - Dynamics: Individual development for the coaching process and leaders' impact on others 
- Career-Competence: Career plan and gaps versus organizational definitions of leadership

\section{Definitions}

The contextual coaching approach is heavily influenced by the definitions of context, executive and organizational coaching that each coach recognizes. Definitional variation is also reflected in the existing literature. Table 1 below provides the most common definitions for each of these elements.

Table 1: Definitions of context, executive and organizational coaching

\begin{tabular}{|c|c|}
\hline Author/Source & Description \\
\hline $\begin{array}{l}\text { (Johns, 2006) } \\
\text { Definition of } \\
\text { Context }\end{array}$ & $\begin{array}{l}\text { "Situational opportunities and constraints that affect the } \\
\text { occurrence and meaning of organizational behavior as } \\
\text { well as functional relationships between variables. } \\
\text { Context can serve as a main effect or interact with } \\
\text { personal variables such as disposition to affect } \\
\text { organizational behavior" (p. 386). }\end{array}$ \\
\hline $\begin{array}{l}\text { (Natale, et al., } \\
\text { 2005) } \\
\text { Executive } \\
\text { Coaching }\end{array}$ & $\begin{array}{l}\text { "that which relates to managerial-executive level } \\
\text { development for performance and professional growth. } \\
\text { The working definition of executive coaching for the } \\
\text { study was: "a collaborative, solutions-focused, results- } \\
\text { oriented and systematic process in which the coach } \\
\text { facilitates the enhancement of performance, self-directed } \\
\text { learning and the personal growth of individuals" (pp. 65- } \\
67) \text {. }\end{array}$ \\
\hline $\begin{array}{l}\text { (Maltbia, et al., } \\
\text { 2014) } \\
\text { Organizational } \\
\text { Coaching }\end{array}$ & $\begin{array}{l}\text { A developmental process that builds a leader's } \\
\text { capabilities to achieve professional and organizational } \\
\text { goals" (p. 165). }\end{array}$ \\
\hline $\begin{array}{l}\text { (Gorrell, et al., } \\
\text { 2009) } \\
\text { Contextual } \\
\text { Coaching }\end{array}$ & $\begin{array}{l}\text { "A system oriented or holistic approach to coaching, that } \\
\text { proposes the incorporation and alignment of certain } \\
\text { contextual elements in the coaching approach increases } \\
\text { the effectiveness of the behavior change in the } \\
\text { organizational setting" (p. 5). }\end{array}$ \\
\hline
\end{tabular}


organizational behavior in context (Johns, 2006, p. 386). The focus of contextual coaching is its reliance on the coach identifying these opportunities and constraints through their interaction during the engagement, incorporating them into the client objective setting and ensuring alignment between the resulting objectives and organizational expectations.

\section{Origins/history}

Although the exact origins of executive coaching are unclear (KampaKokesch, et al., 2001), the process of coaching managers can be traced back to the late 1980s (Tobias, 1996). The origins for organizational or systemsoriented coaching is even more elusive. Paul Gorrell and John Hoover developed their framework of contextual coaching during the 1990s (Gorrell, et al., 2009); however, the concept has only recently received renewed attention. What makes the task of tracing the history of a coaching approach more complicated is the variation in use of terms. For example, three terms that are most associated with organizational coaching: performance coaching, executive coaching, and alignment coaching are often used interchangeably. Although they all refer to work with leaders in their organization with the goal of increasing leader effectiveness, it is not clear to what extent context is a consideration or where overlaps exist. Contextual or systems coaching theorizes that a disciplined approach to aligning the coaching work with organizational objectives will result in enhanced outcomes. To date there has been limited empirical work validating which key elements in context most influence coaching work and those incorporating approaches that highly correlate to enhanced outcomes.

\section{Conceptual Framing}

Regardless of the framework, the consistent theoretical underpinning of context-based coaching is the alignment between the organizational objectives, individual developmental objectives, and coach agenda. In theory, the closer the alignment between these elements the stronger the impact on the overall effectiveness of the investment in the developmental work. This contextual coaching alignment is represented through the overlaps between the coach's approach, the coaching client's developmental objectives, the organizational objectives and expectations surrounding the firm's talent, represented in Figure 1. 


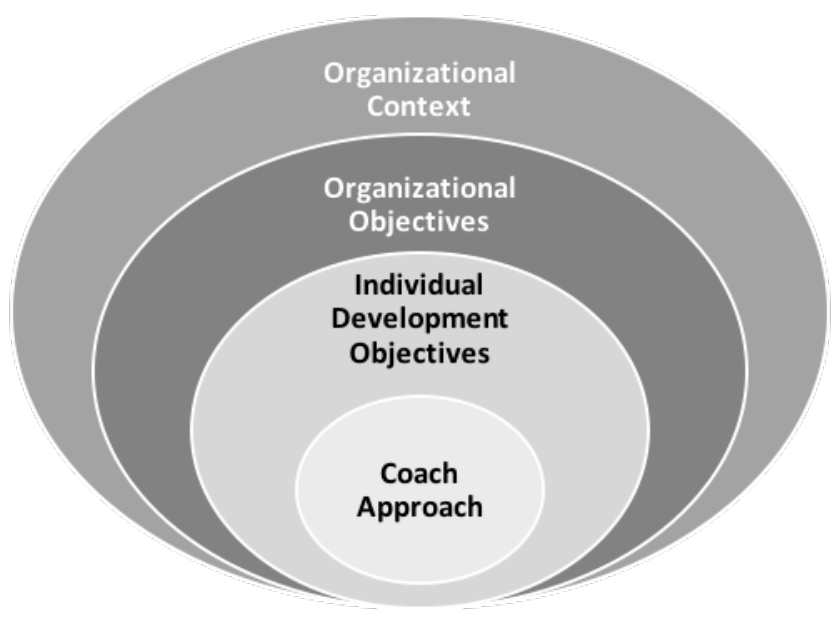

Figure 1: Contextual Alignment Elements

\section{Study Approach}

The complex relationship between the elements in contextual coaching require a wholistic approach to examine it. The multi-phased study therefore employs a mixed method (Qualitative - Quantitative) scheme; it utilized archival data interviews and questionnaires in the first two phases of work, and will use surveys to validate the factors in an organization's context that act as enablers or barriers to behavior change identified by the qualitative data. The study examines the relationship between the incorporation of contextual factors in the coaching approach and the attainment of desired coaching outcomes.

The study was designed to be conducted in three phases. The first two phases incorporated qualitative data and is reported here. The quantitative last phase will be informed by the findings from the qualitative work presented in this paper. Each phase of work is intended to inform the next phase of work. During the qualitative first phase of work, a random review of 50 coaching reports from engagements with organizational leaders or groups was evaluated. These narrative reports, completed by the coach, provided details of the coach's interactions in the organization. Multi-rater feedback and interviews conducted on behalf of the coaching client, the individual objectives set by the coaching client, and perceived barriers or enablers to development identified by the coach, was typical information contained in these reports. The review of the coaching reports was intended to answer two key questions: to what extent did the coach connect engagement objectives to contextual factors, indicating the importance of context to the work, and to what extent did contextual factors 
emerge during the various coaching conversations. The themes identified during the archival review informed interview questions used during the second phase. The data collected from interviews informed a pilot survey design to be used in phase three. Four lines of inquiry were pursued during the interview phase:

- How the coach viewed the importance of context in their coaching work

- What elements of context the coach viewed as the most influential to achieving coaching outcomes

- What elements of context the coach viewed as most influential to achieving sustained behavior change

- How did the coach incorporate context into their coaching work?

Twelve (12) in-person interviews of 40-60 minutes and twenty-seven (27) four-question questionnaires were completed during the second phase of work. A manual thematic analysis (Boyatzis, 1998) was applied to all questionnaires and interview transcripts. Each transcript was coded to identify recurring terms or descriptors and grouped into clusters, the clusters were then validated by an independent reader. A theme was generated from the clusters only after saturation was reached (Strauss \& Corbin, 1998, p. 136) and it appeared in at least $20 \%$ of the interview and questionnaire transcripts. The themes identified through the analysis were incorporated into a 10-question test survey that was sent to a wider distribution of coaches. A random sample of 17 completed surveys were evaluated for face validity. Basic descriptive statistical techniques were utilized to further understand variation in the survey data. This "pilot" survey is intended to inform a future and final phase of work that utilizes correlational analysis to further refine the themes from the qualitative portion of the study.

\section{Findings}

The first two phases of this study provide several key insights associated with understanding the role of context in coaching. First, it validates the importance coaches attach to the influence context has on coaching outcomes. Secondly, it suggests that coaches can identify discrete factors that inhibit or enable outcomes during a coaching engagement and influence the prospect of achieving sustainable developmental change. Finally, the study provides understanding into approaches used by coaches, to incorporate contextual considerations into their work. Each of these insights will now be explored in more detail. 


\section{Importance of Context}

There is a significant variation in how coaches define, recognize, and assess contextual factors. However, there was strong agreement on its importance to achieving coaching outcomes and sustained behavior change by the coaching client. The relationship between how each coach perceives the importance of context to a specific engagement and how they incorporate it into coaching work remains unclear. In some cases, coaches stated context was only mentioned as a mechanism to develop heightened self-awareness about potential challenges to achieving agreed-upon coaching objectives. In other cases, coaches claimed to regularly create strategies with the client, informed by perceived contextual barriers to development. Nevertheless, two consistent themes were identified in the study, that provide additional clarity on this topic:

- Coaches suggested that the level of importance for any contextual factor was dependent on engagement objectives or the developmental need of the coaching client. This suggests that there may not be a universal set of contextual elements that can be applied in all systems.

- Coaches placed a high level of importance on the connection of contextual factors to achieving coaching outcomes but differ on incorporation strategies and indicated a concern that a focus on context will inhibit coaching work by taking focus away from the individual.

\section{Contextual Factors most influential to achieving coaching outcomes:}

Coaches identified several factors that were deemed as most influential on the ability of the coach to achieve desired coaching outcomes.

- Goal orientation of the organization (related to the perceived accountability, on the part of the client, for achieving agreed upon developmental objectives and the perceived degree to which goals and goal accountability are a natural aspect of the existing culture).

- Control orientation or perceived ability by the learner to achieve their agreed upon coaching objectives in the organization. This factor was often associated with the coaching client's perception of how important the coaching objectives were against other organizational priorities and expectations.

- Perception of organizational fairness and inclusivity with regards to advancement and assignments (level of perceived bias in talent decisions). 
- Perceived organizational safety (or encouragement) to take risk in working on new skills or behaviors in public. This is expressed as the perceived reaction to failure, the safe opportunities that would be provided to experiment and the level of encouragement or feedback that could be expected by those who were most important to the coaching client.

- Level of (senior) leadership engagement in the developmental process/activities of the organization across the enterprise and its visibility to the coaching client.

- Organizational developmental mindset. Two dimensions were identified related to the demonstration of a developmental mindset by senior leaders (to what extent are senior leaders perceived to be open to and encourage critical feedback), and the perceived organizational attitude with regards to the efficacy of coaching (who receives coaching, what happens after an engagement, and how coaching work is socialized in the system).

\section{Contextual factors most influential to achieving sustained behavior change by the learner}

Coaches identified several factors that were perceived to have the most influence on the ability of a learner to sustain behavior change resulting from coaching work:

- Level of authentic engagement by the direct supervisor in the coaching process and the presence of accountability for agreed upon objectives.

- Peer pressure to advance in the organization.

- Level of organizational accountability on leader competencies and the connection of coaching work with those competencies.

- The presence of a positive feedback culture in the organization.

\section{Incorporation of context}

Only one theme emerged from the examination of how coaches incorporate context into their coaching work or how they align contextual factors with coaching objectives. 
When discussing incorporation approaches coaches most often referred to activities associated with raising the coaching client's awareness of contextual factors that impede achievement of developmental objectives and the creation of strategies to mitigate perceived impediments.

\section{Overall findings}

The findings suggest that coaches can identify discrete elements in context with disproportional impact on coaching outcomes and they actively consider how to incorporate them into their coaching work. The data suggest that a strong correlation may exist between the visible level of leadership engagement (outside of HR) in the coaching process and the attainment of coaching outcomes by those who participate in the process. This may be complemented by the extent to which the prevailing view of coaching in the organization is positive and the culture is a feedback-oriented culture.

The findings also suggest that the broad descriptions of contextual elements initially positioned in early models may not be as useful for coaches or the organization. This study advocates for more narrow and measurable elements that are closely relevant at the level of interaction than generic elements. While it may be appropriate to provide a high-level structure that allows for comparison across multiple engagements and coaches, the data suggest that proximity to the coaching client's agenda is a key consideration to how contextual factors should be identified and accounted for.

\section{Conclusions and Recommendations}

The first two phases of this study represent an initial stage of validating the influence of context on coaching work and sustained behavior change. It advances the theory that a coach possessing an ability to recognize the factors in the organization's environment that are most relevant to the client's developmental work, and the ability to incorporate and align those factors, will produce higher quality outcomes as compared with coaches who do not possess these skills. Therefore, we conclude the following from the work:

\section{Identification of factors}

- Although definitional challenges with consistency exist, discrete factors in context that support or inhibit the attainment of developmental objectives can be identified by coaches who work in the organizational setting. 
- The importance of a factor may vary according to coaching or developmental objectives, client profile, or other factors.

- Coaches can distinguish those factors that directly influence the ability to successfully achieve coaching outcomes (enabling the conditions for an effective coaching relationship) from the factors that enable sustainable behavior change on the part of the coaching client.

- Ten distinct factors were identified in the study which may be further consolidated into clusters.

\section{Implications for practice}

Clearly refining and developing a measurement protocol for those contextual factors most relevant to coaching effectiveness and sustained behavior change could have implications far beyond increasing the effectiveness of each engagement. Armed with an understanding of how to identify and measure the factors that enable the system to strengthen conditions for development system wide would also provide a mechanism to inform broader strategic development initiatives. Further, firms that use coaching widely have an opportunity to collect data from multiple engagements over time, allowing them to uncover potentially hidden themes and patterns in their culture. From an organizational perspective, then, a systems-based coaching approach provides the following return on investment (ROI) opportunities:

1. The potential for improving the overall developmental environment through the systematic evaluation of contextual measures unique to each organization and by providing a process whereby the organization gains consistent "climate" feedback from each engagement. This systematic approach may provide useful information for developing coaching competency for internal coaches and potentially increase the effectiveness of coach matching for external coaches. The system level contextual measures may also increase the awareness of supervising managers to potential barriers that could derail sustained development of their coached direct report.

2. The potential to link contextual factors to support organizational leadership competency modeling.

3. The potential for more targeted and focused developmental initiative design based on an established contextual framework.

4. Enhancing the consistency of coaching across the enterprise by providing a common set of contextual factors for coaches to integrate 
into their work. This allows the organization to better monitor and align coaching work across the enterprise.

5. The opportunity to enhance each coach's effectiveness through increased awareness of specific contextual information that support or inhibit coaching work in that system. Theoretically a new coach working in the system would have the benefit of the system knowledge obtained by all the prior engagements.

\section{Limitations}

This study has several limitations and opportunities for additional inquiry. The sample size used for this study was small. Only 39 coaches provided their perspective. In addition, the data was collected primarily from coaches practicing in the United States and as such is limited by its lack of global reach. It is suggested that a much larger sample of coaches be invited to provide a narrative perspective on the subject. A second significant limitation is the reliance on the coach-centric perspective to form the findings. While useful to understand incorporation approaches, the reliance on coach-only data subjects the study to self-interest bias with regards to the perceived importance of context in achieving coaching outcomes and realizing sustained change.

These limitations provide potential avenues to expand the work by examining data from the perspective of client population and expanding the sample. A correlational analysis on the factors identified by coaches and the relationship on the ability to achieve coaching outcomes and sustain those outcomes also represent a possible avenue of further study. The study also presents a need for further examination of how to measure each factor's influence on developmental objectives and behavior change sustainability. Additional work in understanding how factor relevance changes with developmental focus or client profile would also be useful for coaches preparing for executive engagements and providing system level insights.

\section{References}

Boyatzis, R. E. (1998). Transforming Qualitative Information. Thousand Oaks: Sage Publications.

Cappelli, P., \& Sherer, P. D. (1991). The missing role of context in OB: The need for a meso-level approach. Research in Organizational Behavior, 55-110. 
Doolen, T. L., Hacker, M. E., \& Van Aken, E. M. (2003). The impact of organizational context on work team effectivness: A study of production team. IEE Transactions on Engineering Management, 285-296.

Eagly, A. H., \& Chaiken, S. (1993). The Psychology of Attitudes. Fort Worth: Harcourt Brace Jovanovich.

Fishbein, M., \& Ajzen, I. (2010). Predicting and Changing Behavior. New York: Psychology Press.

Funder, D. C. (2001). Personality. Annual Review of Psychology, 197-221.

Glasgow, J. M., Yano, E. M., \& Kaboli, P. J. (2013). Impacts of organizational context on quality improvement. American Journal of Medical Quality, 196-205.

Gorrell, P., \& Hoover, J. (2009). The coaching connection: A managers guide to developing individual potential in the context of the organization. New York: American Management Association.

Gregory, B. T., Harris, S. G., Armenakis, A. A., \& Shook, C. L. (2009). Organizational culture and effectivness: A study of values, attitudes and organizational outcomes. Journal of Business Research, 673-679.

Johns, G. (2006). The essential impact of context on organizational behavior. Academy of Management Review, 386-408.

Kampa-Kokesch, S., \& Anderson, M. Z. (2001). Executive coaching: A comprehensive review of the literature. Consulting Psychology Journal: Practice \& Research, 205-228.

Maltbia, T. E., Marsick, V. J., \& Ghosh, R. (2014). Executive and organizational coaching: A review of insights drawn from literature to inform HRD practice. Advances in Developing Human Resources, 161182.

Natale, S., \& Diamante, T. (2005). The 5 stages of executive coaching. Journal of Business Ethics, 361-374.

Parzinger, M. J., Lemons, M. A., \& McDaniel, K. (2012). The impact of organizational context on turnover and job satisfaction: a multi-analysis study of bank employees. International Journal of the Academic Business World, 39-46.

Porter, L. W., \& McLaughlin, G. B. (2006). Leadership and the organizational context: Like the weather. The Leadership Quarterly, 559-576.

Shalley, C. E., \& Gilson, L. L. (2004). What leaders need to know: A review of social and contextual factors that can foster or hinder creativity. The Leadership Quarterly, 33-53.

Sila, I. (2007). Examining the effects of contextual factors on TQM and performance through the lens of organizational theories: An empirical study. Journal of Operations Management, 83-109. 
Strauss, A., \& Corbin, J. (1998). Basics of qualitative research: Techniques and proceedures for developing grounded theory (2nd Ed.). Thousand Oaks: Sage .

Tobias, L. L. (1996). Coaching executives. Consulting Psychology Journal: Practice \& Research, 87-95.

\section{Author Contact}

The author, Mike Valentine, of TruEdge Consulting, can be contacted via email at mike@truedgeconsulting.com. 